\title{
Intensity Spread Analysis of Programmable Photonic Circuits with Parasitics
}

\author{
Iman Zand, Banafsheh Abasahl and Wim Bogaerts \\ Ghent University - imec, Photonic Research Group, Department of Information Technology, Gent, Belgium. \\ Center of Nano and Biophotonics, Ghent University, Belgium. \\ Email: iman.zand@imec.be
}

\begin{abstract}
Small stochastic imperfections in the phase and coupling $(<1 \%)$ of tunable components in programmable photonics circuits introduce unwanted interferences deteriorating their frequency response. Performing Monte-Carlo simulations, we investigate such imperfections for different routings of light through a 7-cells hexagonal mesh with different biasing schemes.
\end{abstract}

Index Terms-Photonics, Programmable Circuits, Parasitics

\section{INTRODUCTION}

Recently, programmable Photonic Integrated Circuits (PICs) have gained a lot of interest [1]-[3] because of their potential to configure various functions in a single circuit. Programmable PICs are basically composed of mesh of waveguides, tunable $2 \times 2$ couplers, and phase shifters, where the connectivity can be defined in both amplitude and phase. Hence, the accuracy of the controlling scheme of tunable $2 \times 2$ couplers and phase shifters, happening in software through an electrical driver circuit, plays an important role. Effects such as nonlinear response curves, electrical and thermal crosstalk, or discretization in digital-to-analog conversion can introduce imperfections in tuning. This introduces parasitic effects such as additional loss, phase errors, and coupling errors, which create secondary and tertiary paths for the light, that can cause unwanted interferences and resonances in the mesh circuit.

\section{Methodology}

For the mesh architecture, we use a 7-cell hexagonal mesh, where the $2 \times 2$ couplers (CPs) are connected by silicon waveguides (Fig. 1a). The coupling coefficient of the CPs can vary between $0-100 \%$ and their arm length is assumed to be $300 \mu \mathrm{m}$. For the simulations, We have extended the design framework IPKISS by Luceda Photonics with an automatic mesh generator, written in python.

To investigate the effect of parasitics on routed paths through the configured meshes, an intensity spread analysis has been done using Monte-Carlo cycles with 100 samples, applying a normally distributed variation in the coupling $\kappa$ of all CPs with different standard deviations. This results in a set of transmission spectra (Fig. 1b-c) for different mesh configurations. We assess the intensity

This work has received funding from the European Unions Horizon 2020 research and innovation programme under grant agreement No 780283 (MORPHIC) from the European Research Council under grant agreement No 725555 (PhotonicSWARM).

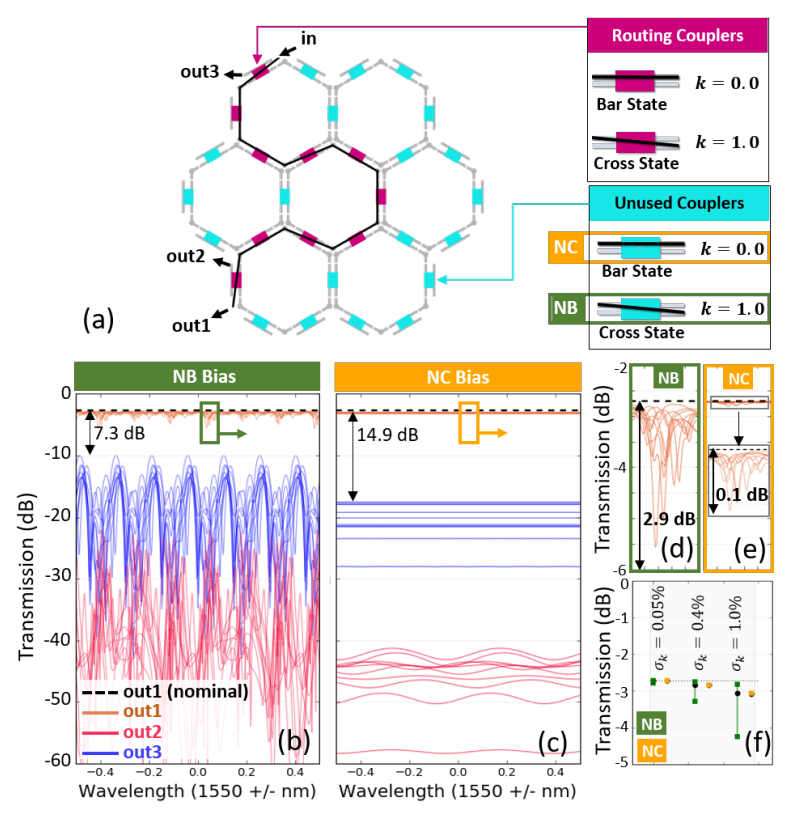

Fig. 1. a) Schematics of a routed path (with $L_{\text {path }}=10 L_{u}$ ) within a 7cells Mesh configured for two types of biasing: a) Normally-Bar (NB): unused couplers (blue) are biased in bar state and b) Normally-Cross (NC): unused couplers biased in cross state. b,c) Transmission spectra of the mesh for these two types of biases. e,f) Scaled windows of the transmission spectra near 1.55 $\mu \mathrm{m}$. g) Intensity spread analysis of transmission in out 1 for random variations of $\sigma_{k}=0.05 \%, 0.4 \%, 1.0 \%$, green and yellow error bars correspond to the $\mathrm{NB}$ and NC biases.

spreads with error bars, where [min, mean, max] points are obtained by: $\min \left(\left[\min (T(\lambda))_{5}, \ldots, \min (T(\lambda))_{95}\right]\right)$, $\operatorname{mean}\left(\left[\operatorname{mean}(T(\lambda))_{5}, \ldots, \operatorname{mean}(T(\lambda))_{95}\right]\right), \quad$ and $\max \left(\left[\max (T(\lambda))_{5}, \ldots, \max (T(\lambda))_{95}\right]\right)$. The subscript 5 and 95 indicate that we only considered the 5-95th percentile of the samples, discarding the most extreme values.

To route light between two desired ports, couplers are only programmed in either cross state $(\kappa=1)$ or bar state $(\kappa=0)$. In this process, some of the couplers are used to create a route which are referred as Routing Couplers (RCPs). On the other side, there are some Unused Couplers (UCPs) which are not involved in the routing and their state does not change the path shape. For these, we have used two different biasing schemes: setting all the UCPs in 1) bar state or 2) cross state. We refer to these biases as "Normally Bar" (NB) and "Normally Cross" (NC) (Fig. 1a). 


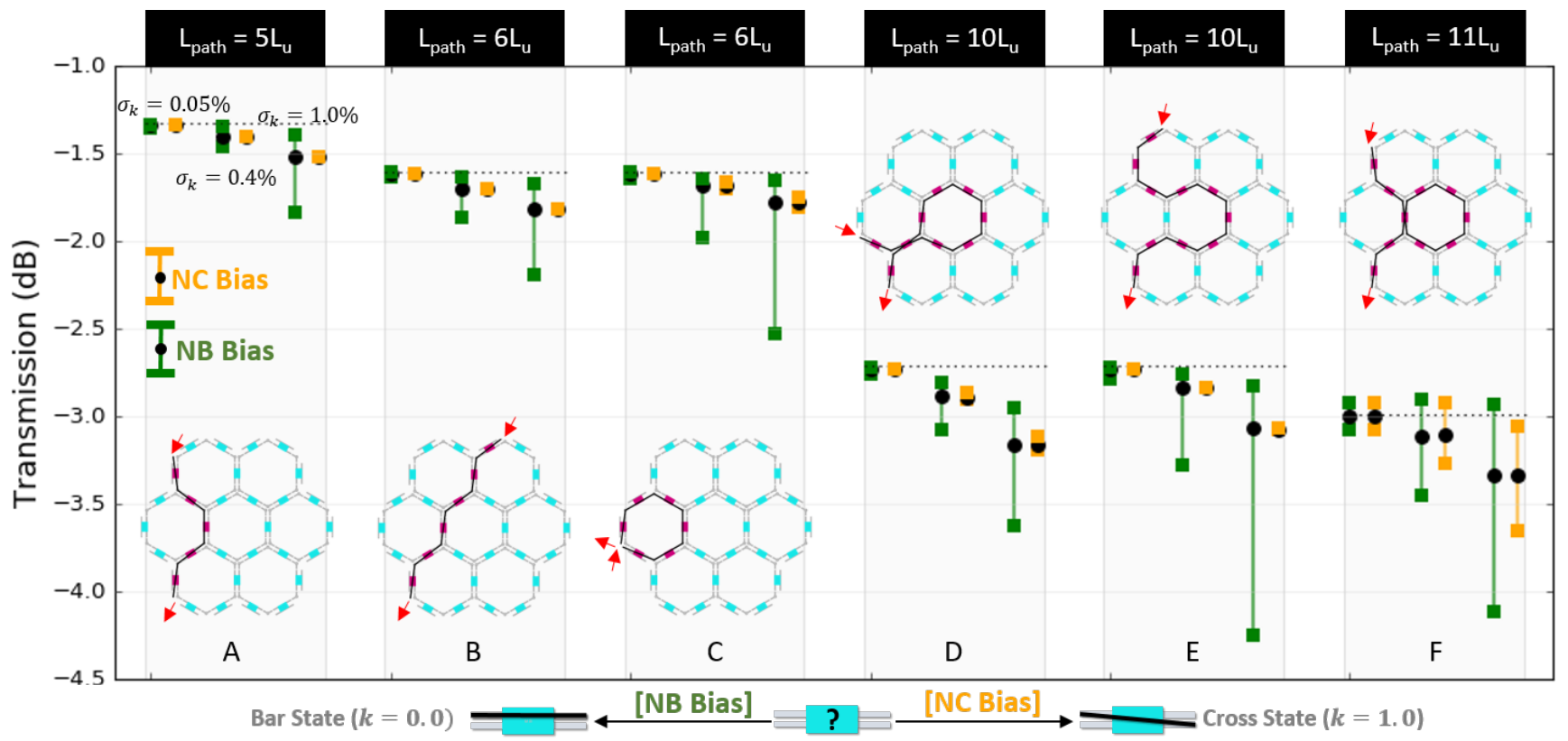

Fig. 2. Intensity spread analysis of different configurations of the 7-cells mesh to study both simple and complex paths. Blue couplers are in the Bar (Cross) state for the NB (NC) bias. Random variations of $\sigma_{k}=0.05 \%, 0.4 \%, 1.0 \%$ are chosen for the Monte-Carlo simulations. Green and yellow error bars correspond to the NB and NC bias, respectively.

\section{RESULTS}

To show the effect of NB and NC biases on the parasitic circuits, the transmission spectra of a path ( with $L_{\text {path }}=10$ $L_{u}$ ) routed through a 7-cell mesh are plotted in Fig. 1b-c, using only 10 Monte-Carlo cycles with $\sigma_{k}=1.0 \%$ of the couplings $\kappa$. For NB bias, UCPs (indicated by blue couplers in Fig. 1a) are set to be in bar state and, for the NC bias, they are set to be in cross state, but in both cases with a small error provided by the Monte-Carlo simulation. Comparing the levels of the transmissions with those of the ideally programmed mesh (black dashed lines) shows that, for both bias types, the unwanted couplings will introduce losses because light is tapped out of the main path. In addition, at the desired output port (out1) we see significant ripples of the transmission spectra in NB bias (caused by parasitic interference paths), which are strongly suppressed in the NC bias. Fig. 1d-e clearly show this effect, where ripples with depth of $2.9 \mathrm{~dB}$ are decreased to $0.1 \mathrm{~dB}$. We see ripples for light coming out of unwanted ports (out2,out3). This significant improvement of the intensity spread for NC bias suggests that setting all UCPs to the cross state reduces light accumulation in the circuit, suppressing resonances in parasitic coupled ring resonators. For a meaningful stochastic analysis, the intensity spreads of the transmission responses are calculated for both types of biases, as shown in Fig. 1f, where 100 cycles of Monte-Carlo and random variations of $\sigma_{k}=0.05 \%, 0.4 \%, 1.0 \%$ are applied. Clearly, NC bias reduces the intensity spread of transmission for more than $95 \%$.

Next, we investigate effect of both biasing schemes on paths with simple and complex routings. Fig. 2 shows the intensity spread analysis of several paths similar to the one presented in Fig. 1f. In NB bias, larger $\sigma_{k}$ causes more loss and intensity spread (deeper ripples in spectrum). It is also seen that complex paths with a loop in their configuration $(\mathrm{F})$ can have higher transmission than the nominal response; in fact, parasitic shortcuts (shorter paths with lower loss than the main path) are prominent and result in constructive interference of the output signal arriving through shortcuts. On the other side, for the $\mathrm{NC}$ bias, parasitics interferences are almost eliminated for the simple paths and the intensity spread is almost reduced to zero.

\section{CONCLUSION}

Two biasing schemes for the unused couplers in a hexagonal 7-cell mesh are compared on their impact on parasitic paths: The unused couplers are set in Bar state (NB bias) or in Cross state (NC bias). Monte-Carlo simulations show that NC bias considerably suppresses the effects of parastics on transmission response of the circuit for paths with different routing.

\section{REFERENCES}

[1] D. A. B. Miller "Self-configuring universal linear optical component," Photon. Res., vol. 1(1), pp. 1-15, (2013).

[2] D. Pérez, I. Gasulla, L. Crudgington, D. J. Thomson, A. Z. Khokhar, K. Li, W. Cao, G. Z. Mashanovich, and J. Capmany, "Multipurpose silicon photonics signal processor core," Nature Comm., vol. 8(636), pp. 1-9 (2017).

[3] D. Pérez, I. Gasulla, and J. Capmany, "Programmable multifunctional integrated nanophotonics - Review Article," De Gruyter - Nanophotonics, vol. 7, pp. 1351-1371 (2018). 